\title{
Análisis de la situación en la pista de los jugadores en el saque y su relación con la dirección, el lado de la pista y el resultado del punto en pádel de alto nivel Analysis of the situation on the court of the players in the serve and its relationship with the direction, the side of the court and the result of the point in high-level padel
}

\author{
*Jesús Ramón-Llin, *José Guzmán, *Rafael Martínez-Gallego, **Diego Muñoz, ***Alejandro Sánchez-Pay, \\ ***Bernardino Javier Sánchez-Alcaraz \\ *Universidad deValencia (España) **U niversidad de Extremadura (España) ***U niversidad de M urcia (España)
}

\begin{abstract}
Resumen. El objetivo deeste estudio fue conocer la influenciade lasituación en la pista de los jugadoresal saquey su relación con la dirección, el lado de la pistay el resultado del punto en pádel de al to nivel. La muestra incluyó un total de 1071 puntos correspondientes a nueve partidos donde participaron 36 jugadores masculinos de 10 Categoría Nacional. Se analizaron variables relacionadas con la tácticautilizada en el saque (tradicional o australiana), la dirección del saque (pared lateral 0 «T»), el lado de la pista (derecho o izquierdo) y el resultado del punto (gana o pierde la pareja al saque). Los resultados mostraron que losjugadores sacan entre un $75 \%$ y un $85 \%$ de las ocasiones a la pared lateral en el lado de izquierdo de la pista. En el lado derecho, se incrementa el porcentaje de saques a la «T», especialmente utilizando una situación en la pista australiana. A nivel general, losjugadores ganan un porcentaje mayor de puntosal saque cuando utilizan latácticatradicional frenteala australiana. Sin embargo, estas diferencias no fueron significativas cuando los jugadores sacar on al a «T» 0 al la par ed lateral, desde ambos lados de la pista. Finalmente, el porcentaje de puntos ganados al saque disminuyó a medida que avanzó el partido de pádel, especialmente con táctica australiana del segundo al tercer set. Estos datos tienen una importante aplicación práctica en el diseño de ejercicios de pádel basados en las demandas técnico-tácticas específicas de la competición.
\end{abstract}

Palabras clave: Deportes de raqueta, análisis del rendimiento, acciones de juego, servicio, competición.

\begin{abstract}
The aim of this study was to know the influence of the players' serve tactical position and its relationship with the direction, the side of the court and the point outcome in elite padel. The sample included a total of 1071 points corresponding to nine matches with 36 male players of 1st National Category. Variables related to the serve tactic position (conventional or Australian), serve direction (side wall or «T»), court side (right or left) and point outcome (serve pair win or lose) were analyzed. The results showed that padel players serve between $75 \%$ and $85 \%$ of the times to the side wall on the left side of the court (advantages side). $0 \mathrm{n}$ theright side, the percentage of serves to the «T » isincreased, especially using anA ustralian tactical position. Furthermore, serving pair win a higher percentage points when they usetraditional tactic. However, these differences are not significant when the players serve to the «T» or the side wall, from both sides of the court. Finally, the percentage of points won by serving pair decreases as the padel match progresses, especially with Australian tactic from the second to the third set. These data have an important practical application in the design of padel exercises based on the specific technicaltactical competition demands.
\end{abstract}

Key words: Racket sports, performance analysis, game actions, service, competition.

\section{Introducción}

El pádel es un deporte de raqueta, nacido en México hace aproximadamente 50 años (Sánchez-Alcaraz, 2013), que ha experimentado un enorme crecimiento en la última década tanto en el número de jugadores como de instalaciones deportivas (Courel-Ibáñez, SánchezAlcaraz, García, et al., 2017; M uñoz, Sánchez-Alcaraz, et al. , 2016), practicándose en más de 40 países al rede-

Fecha recepción: 21-09-20. Fecha de aceptación: 07-01-21 PreferenciasAlejandro Sánchez-Pay aspay@um.es dor del mundo (Federación Internacional dePádel, 2020). Las investigaciones en pádel se han centrado principalmente en describir los parámetros de la competición y detectar indicadores de rendimiento efectivos (CourelIbáñez et al., 2015, 2019; Torres-Luque et al., 2015), y se han basado principal mente en cuatro ár eas fundamentales: aspectos temporales (Courel-Ibáñez, SánchezAlcaraz, \& Cañas, 2017; Courel-lbáñez \& SánchezAlcaraz, 2017; Muñoz, García, et al., 2016; Pradas et al., 2014), movimientos de los jugadores y distancias recorridas en la pista (Amieba \& Salinero M artín, 2013; Priego et al., 2013; Ramón-Llin et al., 2014, 2021), acciones de juego (Courel-Ibáñez et al., 2019; García 
Benítez, Pérez-Bilbao, Echegaray, \& Felipe, 2016; M uñoz et al. , 2017; Torres-Luque et al. , 2015) y variables fisiológicas como la frecuencia cardiaca (M artínez, M oreno \& Verdú, 2017; Ramón-Llin, Guzmán \& Martínez-Ga Ilego, 2018) 0 antropométricas (Castillo-Rodríguez, Hernández-M endo \& Alvero-Cruz, 2014; M uñoz, ToroRomán, Grijota, Courel-Ibáñez, Sánchez-Pay \& SánchezAlcaraz, 2021; Zabala et al., 2016). Los resultados de estos estudios confirman que estos indicadores de rendimiento varían en función de distintas variables como el género y el nivel de los jugadores (Courel-Ibáñez et al., 2019; Tor res-Luque et al., 2015).

Sin embargo, aunque se han realizado un importante número de estudios centrados en el análisis de la competición en pádel profesional, hay una alarmante escasez de trabajos que analicen las estadísticas relacionadas con el saque y el resto en padel (Ramón-Llin et al., 2019). El saque o servicio, ha sido considerado como el indicador de rendimiento más importante en los deportes de raqueta (Gillet et al., 2009). En tenis, el servicio otorga la posibilidad de ganar directamente el punto a través de un saque directo o indirectamente tomando la iniciativa del punto sobre el oponente a tra vés de un buen servicio (Reid et al., 2010). De este modo, los jugadores de tenis ganan aproximadamente un $70 \%$ de los puntos con el primer servicio, y este porcentaje es mayor en hombres comparado con las mujeres (Gillet et al., 2009; O 'D onoghue, 2001). Estudios previos han mostrado que el servicio es todavía más determinante en la modalidad de dobles, debido a la reducción del espacio para devolver el resto, condicionada por el compañero del sacador que se encuentra en la red, tal y como ocurre en pádel (Furlong, 1995, Martínez-Gallego, Crespo, Ramón-Llin, Micó y Guzmán, 2020) donde también ganan un mayor porcentaje de puntos al servicio los hombres que las mujeres(Carboch, 2016). Por lo tanto, el saque podría tener una influencia todavía mayor en pádel que en tenis, ya que permite al jugador que lo real iza al canzar la posición ofensiva de la red antes que sus oponentes, con la correspondiente ventaja que esto supone (los puntos conseguidos en la red representan alrededor del $80 \%$ del total, y los ganadores consiguen un 34\% más de puntos que los perdedores en esta zona ofensiva) (CourelIbáñez et al. , 2015; Ramón-Llin et al., 2013). En este sentido, un reciente estudio publicado en pádel ha mostrado como la pareja al saque tuvo una ventaja significa tiva en el punto, que se mantuvo hasta el séptimo golpe en mujeres y hasta el doceavo golpe en hombres (Sánchez-Alcaraz et al., 2020).
Sin embargo, las características del servicio en pádel son diferentes a las del tenis, ya que las normas dictan que este debe efectuarse golpeando la pelota, tras un bote en el suelo, por debajo de la altura de la cintura (International Padel Federation, 2020b). Por lo tanto, la bola no puede ser golpeada tan fuerte como en tenis ni con el mismo efecto, lo que podría influir en que la ejecución del resto se realice en unas mejores condiciones (Ramón-Llín et al., 2019). El reglamento dictaminaque el golpe del saque debe realizarse diagonal mente, detrás de la línea de servicio, cambiando de lado tras cada punto (Federación Internacional de Pádel, 2020).

El compañero del sacador no tiene una regla fijada sobre su posición en la pista antes del saque, aunque por regla general suele ocupar las zonas cercanas a la red (Courel-Ibáñez et al., 2019). En este sentido, los juga dores que sacan han adoptado dos tipos de posiciones tácticas (tradicional y australiana). La posición tradicional muestra al compañero del sacador al lado contrario. En la posición australiana, el compañero del sacador se sitúa en el mismo lado de su compañero (Figura 1). Ambas posiciones tienen implicaciones tácticas cuyo principal objetivo es el de ocupar y mantener durante el juego el lado del que cada jugador es especial ista. Sin embargo, el jugador que resta lo hará bajo la presión de tener que dirigir la pel ota a una zona adecuada, variando altura y dirección de los golpes, para dificultar el posterior golpe de la pareja contraria, que se encuentra yaen lared (Lupo et al., 2018; Ramón-Llín et al., 2019). Pese a que la secuencia saque-resto en el comienzo del punto en pádel profesional parece fundamental y decisiva para incrementar la posibilidad de ganarlo (SánchezAlcaraz et al., 2020), apenas hay estudios hasta la fecha que analicen la táctica del saque y su influencia en el resultado final del punto. Por lo tanto, conocer el rendimiento de los jugadores cuando sacan será extremada mente útil para optimizar su táctica durante la competición, y proporcionará una información muy importante a los entrenadores en el diseño de ejercicios y la aplicación de feedbaks (Butterworth et al., 2013). De este modo, el objetivo de este estudio fue analizar la influencia de la situación en la pista de los jugadores al saque, su relación con la dirección, el lado de la pista y el resultado en el pádel profesional.

\section{M etodología}

\section{Participantes}

La muestra incluyó un total de 1071 puntos correspondientes a nueve partidos del cuadro final de 1 a cate- 
goría federada de tres torneos de pádel de categoría nacional. Los participantes fueron 36 jugadores semiprofesionales masculinos, con una edad media de 33.3 \pm 6.9 años. Se redactó un informe del estudio, que todos los jugadores, y la organización firmaron par el uso de las grabaciones de video con fines anónimos y científicos. El estudio se realizó acorde a la Declaración de Helsinki de 2013.

\section{Variables}

Se analizaron variables relacionadas con la táctica utilizada en el saque, tomando como variable independiente la situación en la pista de los jugadores al saque, siendo la dirección del saque, el lado de la pista, el resultado del punto las variables dependientes.

- Situación en la pista de los jugadores en el saque: Siguiendo a Ramón-Llin et al. (2019), la pareja al servicio podía utilizar dos tipos de posiciones tácticas en el inicio del punto:

- Posición tradicional: En este tipo de estrategia, el compañero del sacador se coloca en el lado contrario de la pista desde el que se realiza el saque (Figura 1, pista de la izquierda).

o Posición australiana: En este tipo de estrategia, el compañero del sacador se coloca en el mismo lado de la pista desde el que se realiza el saque (Figura 1, pista de la derecha).

- Dirección del saque: Se utilizó la clasificación propuestapor Sánchez-Alcaraz et al. (2020), distinguiendo entre saques dirigidos a la pared lateral y saques dirigidos a la «T».

- Lado de la pista: Se registró el lado de la pista desde donde se produjo el saque, clasificándose en lado derecho y lado izquierdo.

- Resultado del punto: Se registró el resultado final del punto, agrupándose en función de si lo ganaba la pareja al saque o la pareja al resto.

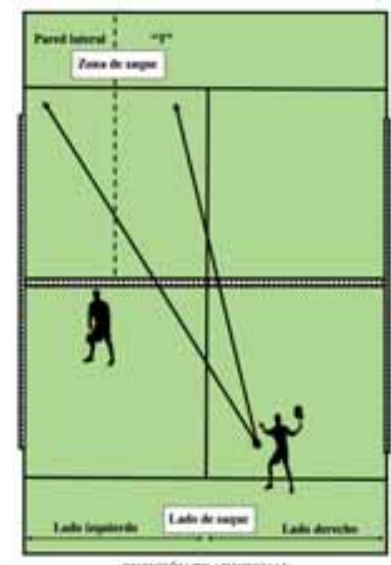

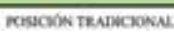

Figura 1. D elimitación de la dirección del saque (pared lateral o "T") y lado de juego (derecho e izquierdo) en la posición tradicional (pista de la izquierda) y austral iana (pista de la derecha).

\section{Procedimiento}

En primer lugar, se solicitó un consentimiento informado a los organizadores de los torneos y a los deportistas para la grabación de los partidos. Los torneos se disputaron en pista cubierta. Los partidos se jugaron siguiendo el reglamento de la Federación Internacional de Pádel (2020). Los partidos fueron grabados utilizando dos cámaras de video digitales modelo Bosch Dinion IP 455 (Bosch, Munich, Alemania), que registran con una frecuencia de 25 fotogramas por segundo. Los datos fueron registrados mediante observación sistemática, a través del uso del software específico LINCE (Gabin, Camerino, Anguera, y Castañer, 2012). Dos observadores Graduados en Ciencias de la Actividad Física y el Deporte, entrenadores de pádel, con más de 5 años de experiencia en el deporte, fueron entrenados específicamente para esta tarea. El entrenamiento consistió en la clara identificación de las variables estudiadas: situación en la pista de los jugadores en el saque, dirección del saque, lado de la pista y resultado del punto. Al término del proceso de entrenamiento, cada observador analizó los mismos 200 puntos con el objetivo de calcular la confiabilidad inter-observador a través del Multirater Kappa Free (Randolph, 2005), obteniendo val ores por encima de . 80 en todas las variables analiza das. Para asegurar la consistencia de los datos, se evaluó la confiabilidad intra-observador al final del proceso de observación, obteniendo valores mínimos de .80. Siguiendo a Altman (1991, p.404), los valores de kappa obtenidos permitieron considerar el grado de acuerdo como muy alto $(>.80)$.

\section{Análisis estadístico}

En primer lugar, se calcularon los estadísticos descriptivos que incluyeron frecuencias y porcentajes. Para realizar la comparativa entre las diferentes posiciones tácticas empleadas por los jugadores al saque y su rela ción con el porcentaje de puntos ganados, el lado de la pista y la dirección del saque se utilizó la prueba chicuadrado de Pearson, con pruebas Z posteriores de comparación de proporciones de columna con ajuste de la significatividad según Bonferroni. Los residuostipificados corregidos (RTC) se calcularon para analizar la significatividad de estas relaciones (Field, 2018). El tamaño del efecto se calculó a partir de la V de Crammer, donde valores de 0.1 representaron un efecto pequeño, 0.3 un efecto mediano y a partir de 0.5 un efecto grande (Fritz et al., 2012). Finalmente, se realizó un calculó de correlaciones a través de la prueba de R ho de Spearman para conocer la relación entre el porcentaje 
de puntos ganados por la pareja al saque en los diferentes sets del partido, en función de si utilizaban una situación en la pista tradicional o australiana. Se estableció un nivel de significación de $p<.05$. Todos los datos fueron analizados con el paquete estadístico IBM SPSS 20.0 para Macintosh (Armonk, NY: IBM Corp).

\section{Resultados}

La tabla 1 muestra diferencias significativas en la dirección del saque en función de la situación en la pista empleada por los jugadores (australiana o tradicional) tanto en los puntos disputados en el lado derecho de la pista $\left(\div^{2}=61.68 ; g l=1 ; p<.01 ; V=.20\right)$ como en el lado izquierdo $\left(\div^{2}=18.32 ; g \mid=1 ; p<.01 ; V=.19\right)$. De este modo, se observa que los jugadores profesionales en pádel, en el lado izquierdo de la pista, sacaron casi un $75 \%$ de las ocasiones a la pared lateral en posición australiana, y este porcentaje fue significativamente mayor en posición tradicional, superando el $85 \%$. Por otro lado, en el lado derecho de la pista, aunque se realizó un mayor porcentaje de saques a la «T» en relación con el lado izquierdo, los jugadores que sacaban en una posición tradicional realizaron un porcentaje significativamente mayor (23.9\%) de saques a la pared lateral cuando adoptan una posición australiana.

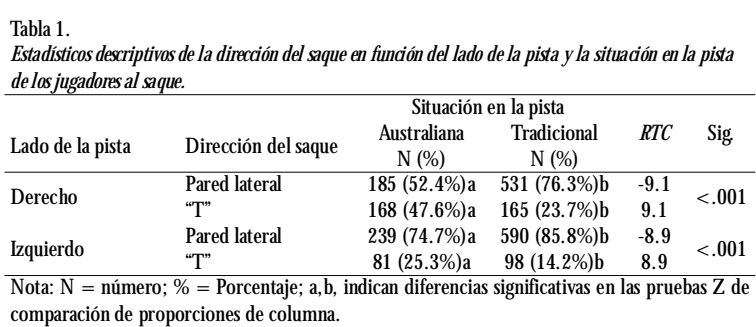

La figura 2 muestra el porcentaje de puntos ganados por la pareja que saca en función de la estrategia utiliza da durante el saque y su relación con el lado de la pista y la dirección del saque. Como se puede observar, en el lado derecho de la pista, los jugadores al saque ganaron entre un 55 y un $59 \%$ de los puntos disputados, independientemente de la dirección del saque y la situación en la pista utilizada. En este sentido, no se observaron diferencias significativas en el porcentaje de puntos ga nados al saque entre la situación en la pista tradicional y la australiana cuando los jugadores sacaron a la «T» $(\div 2$ $=.125 ; g \mid=1 ; p=.724) 0$ a la pared lateral $(\div 2=$ $.093 ; g l=1 ; p=.761)$. Por otro lado, al sacar desde el lado izquierdo de la pista, los jugadores ganaron casi un 8\% más de puntos cuando sacaban en dirección ala «T» utilizando una táctica tradicional frente a la australiana, aunque estas diferencias no fueron significativas $(\div 2=$ $.1130 ; g l=1 ; p=.288)$. Tampoco se encontraron diferencias significativas al sacar a la pared lateral utilizando una táctica tradicional 0 australiana $(\div 2=1537 ; \mathrm{gl}=$ $1 ; p=.464)$.

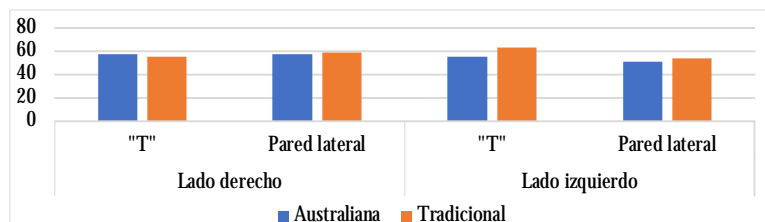

Figura 2. Porcentaje de puntos ganados por la pareja al saque en función de la situación en la pista utilizada (tradicional o australiana), el lado de juego (derecho o izquierdo) y la dirección del saque ("T" 0 pared lateral).

Finalmente, la figura 3 muestra el porcentaje de puntos ganados por la pareja al saque en los diferentes sets del partido, en función de si utilizaban una situación en la pista tradicional 0 australiana. Como se puede observar, en cada uno de los tres sets de los partidos, los jugadores que utilizaban una situación en la pista tradicional ganaron un porcentaje mayor de puntos al saque que aquellos que utilizaron una situación en la pista australiana. Por otro lado, aunque el análisis de correlaciones no mostró una relación significativa entre el número de set y el porcentaje de puntos ganados al saque al utilizar una posición tradicional ( $p>.05$ ) 0 australiana $(p>.05)$, si se observó una disminución del porcentaje de puntos ganados al saque conforme avanza el partido de pádel. En este sentido, los jugadores que utilizan una situación en la pista australiana, perdieron un $6 \%$ más de puntos al saque en el set definitivo del partido que en el segundo set.

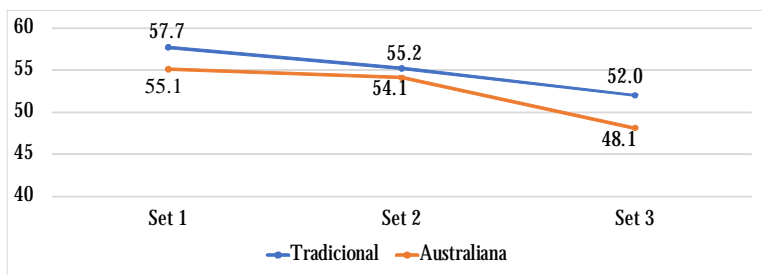

Figura 3. Evolución del porcentaje de puntos ganados al saque durante el partido en función de la situación en la pista utilizada (tradicional o australiana).

\section{Discusión}

El objetivo de este estudio fue conocer la influencia de la situación en la pista de los jugadores al saque y su relación con la dirección, el lado de la pista y el resultado del en pádel semi-profesional. Los resultados de esta investigación mostraron que los jugadores de pádel, desde el lado izquierdo de la pista, sacaron casi un 75\% de las ocasiones a la pared lateral en posición australiana, y este porcentaje fue significativamente mayor en posición tradicional, superando el 85\%. La mayor dis- 
tancia recorrida por el sacador durante el punto ( $R$ a món-Llín et al., 2013), podría explicar esta dirección cruzada del saque al cristal, ya que le permitiría obtener más tiempo para ocupar una mejor posición en la red. Además, el bote en la pared lateral puede dificultar el golpe del restador, lo que podría provocar un mayor número de errores, tal y como afirman otros autores (Lupo et al., 2018).

Siguiendo a Courel-Ibáñez \& Sánchez-Alcaraz (2018), el elevado porcentaje de saques a la pared lateral en el lado izquierdo podría explicarse por la lateralidad de los jugadores. En este sentido, la mayoría de jugadores de pádel que juegan en el lado izquierdo son diestros, por lo que los sacadores tratarían de dirigir la pelota a la pared lateral, hacia el revés del restador, buscando una mayor dificultad en el resto debido al rebote en la pared y el gol peo por parte del restador por su lado no dominante (revés). Por otro lado, en el lado derecho de la pista, aunque se realizó un mayor porcentaje de saques a la «T» en relación con el lado izquierdo, los jugadores que sacaban en una posición tradicional realizaron casi un $24 \%$ más de saques a la pared lateral. Este incremento de saques en dirección a la «T» realizado por los jugadores que adoptan la estrategia austra liana puede ser debido a la intención del sacador de cerrar ángulos en el resto (evitando una devolución paralela) y cubrir mejor el centro de la pista (Ramón-Llin et al., 2019). Este resultado de porcentaje de saques a la pared lateral en el lado de la derecha coincidiría con los resultados indicados por Fernándes de 0 ssó (2019) en categoría masculina tanto amateur como profesional.

Con respecto al porcentaje de puntos ganados al sa que no se observaron diferencias significativas en el porcentaje de puntos ganados al saque entre la situación en la pista tradicional y la australiana cuando los jugadores sacaron a la «T» 0 a la pared lateral. Sin embargo, en el lado izquierdo de la pista, los jugadores ganaron entre un 3 y un $8 \%$ más de saques utilizando táctica tradicional. De este modo, parece que, en el lado izquierdo, utilizar una táctica tradicional podría suponer una ventaja para la consecución del punto. Estas diferencias pueden ser debidas a que el uso de la táctica tradicional podría permitir al sacador situarse más cerca de la red y de la pared lateral en el momento del resto. Aunque esto es una hipótesis que aún no se ha estudia do, concuerda con resultados demostrados estudios previos, golpear en posiciones más cercanas en la red aumentan las opciones de ganar el punto (Sánchez-Alcaraz, Jiménez, M uñoz, Ramón-Llin, 2020, in press).

Por otro lado, teniendo en cuenta el momento del partido, los resultados mostraron cómo los jugadores ganaron un porcentaje mayor de puntos utilizando táctica tradicional frente australiana en cada uno de los sets de los partidos. Además, se observó una disminución del porcentaje de puntos ganados al saque conforme avanza el partido de pádel, especialmente con táctica australiana, donde jugadores perdieron un $6 \%$ más de puntos al saque en el set definitivo del partido que en el segundo set. En este sentido, la fatiga acumulada a lo largo del partido o la presión de tener que remontar 0 mantener la ventaja en el marcador en los periodos finales pueden podrían ser algunos de los factores que influyan negativamente al rendimiento del deportista (Castellar, Pradas, Q uintas, Arraco \& Pérez, 2015, Crespo \& Reid, 2007; Mesagno, Geukes, \& Larkin, 2015).

En comparación con otros deportes de raqueta, existen escasos estudios que analicen la efectividad de las estrategias al saque en modalidades de dobles. En este sentido, en tenis M artínez-Gallego et al. (2019) indica ron una mayor importancia del saque en tenis dobles, pero sin realizar una comparativa de la eficacia de las trayectorias y efectos del servicio como investigaron Gillet et al (2009). Estos últimos autores reportaron mayor eficacia del saque plano para realizar golpes ganadores en dirección a la «T» y mayor eficacia de los saques liftado y cortado en dirección a la esquina abierta. En el caso del bádminton dobles, las estrategias al servicio difieren mucho, puesto que la pareja restadora gana un mayor porcentaje de puntos, especialmente cuando se anticipa el saque corto frente al largo (Gawin, Beyer, Hasse, y Büsch, 2013).

Aunque este es el primer estudio que analiza la situación en la pista de la pareja sacadora en el pádel semiprofesional, el estudio presenta al gunas limitaciones que deben ser tomadas en cuenta a la hora de interpretar los resultados. En primer lugar, no se han analizado algunas variables contextuales como el tiempo de juego 0 el nivel de disputa del partido. Por lo tanto, y debido a la influencia del marcador en la toma de decisiones, especial mente cuando se trata de momentos críticos 0 decisivos (Gómez et al., 2003), se sugiere que futuros trabajos incluyan este análisis. Además, no se han tenido en cuenta tampoco otros datos importantes que pueden afectar a las estadísticas de saque y resto, como la velocidad o el efecto del saque y la lateralidad de los jugadores (C ourel-Ibáñez \& Sánchez-Alcaraz, 2018; 0 'Donoghue \& Ballantyne, 2008; 0 'Donoghue \& Brown, 2009) por lo que se sugiere también su análisis en futuros estudios. 


\section{Conclusiones}

El presente estudio es pionero en analizar la efectividad de diferentes estrategias utilizadas en el saque en pádel, y se pueden establecer diferentes aplicaciones prácticas en el diseño de ejercicios para el entrenamiento y la competición. Los resultados de este estudio señalan que para mantener la efectividad con situación austra liana y estándar al saque, los jugadores cuando saquen al lado izquierdo de la pista deben sacar entre un 75 y un $85 \%$ de veces hacia la pared lateral, mientras que en el lado derecho cuando utilicen una situación australiana en pista deben equilibrar los saques hacia la «T» con respecto a los de pared lateral. Estos resultados también se aplican de igual modo al entrenamiento del resto en pádel. Por otro lado, aunque no se observaron diferencias significativas los resultados indicaron un menor porcentaje de punto ganados al saque usando la situación en pista australiana. Finalmente, el porcentaje de puntosganadosal saque disminuyó a medidaque avanza el partido de pádel, especialmente con la situación en pista australiana del segundo al tercer set, lo que puede indicar una fatiga en el uso de esta estrategia, siendo adecuado ejercicios de preparación física al respecto.

\section{Referencias}

Amieba, C., \& Salinero Martín, J. (2013). Aspectos generales dela competicióndel pádel ysusdemandasfisiológicas AGON International Journal of SportSciences, 3(2), 60-67.

Butterworth, A., O'Donoghue, P., \& Cropley, B. (2013). Performance profilingin sportscoaching: areview. International Journal of PerformanceAnalysisin Sport, 13(3), 572-593.

Castellar, C., Pradas, F., Q uintas,A.,Arraco, S., \& Pérez, J. B. (2015). Perfil condicional dejugadorasdepádel deélite RevistaAndaluza de Mediana De D Eeporte, 8(4), 185-185.

https/ / doi.org/ 10.1016/ j.ramd.2015.08.004

Castillo-Rodríguez,A., Hernández-M endo,A., \&Alvero-Cruz,J. R. (2014). Morfologíadel jugador deélitedepádel: comparación con otrosdeportesderaqueta International Journal of M orphology, 32(1), 177-182. https/ / doi.org/ 10.4067/ \$0717-95022014000100030

Carboch, J., \& Koèib, T. (2016). A comparison of service efficiency between players of maleand femaledoublesat professional tennis tournaments. Acta U niversitatisCarolinae:Kinanthropologica, 51(2), 56-62. https/ / doi.org 10.14712/ 23366052.2015.32

Courel-Ibáñez, J., \& Sánchez-Alcaraz, B. J. (2017). Efecto delasvariz blessituacionalessobrelospuntosenjugadoresdepádel deélite. Apunts Educacion Fisica y Deportes, 127(1), 68-74. https/ / doi.org/ 10.5672/ apunts.2014-0983.es (2017/ 1).127.07

Courel-Ibáñez, J., \& Sánchez-Alcaraz, B. J. (2018). The role of hand dominancein padel: performanceprofilesof professional players.
Motricidade, 14(4), 33-41. https:/ / doi.org/ 10.6063/ motricidade 14306

Courel-lbâñez,J., Sánchez-Alcaraz, B.J., \& \& Cañas,. (2015). Effectiveness tt thenet asapredictor of final match outcomein professional padel players. International Journal of PerformanceAnalysisin Sport, 15(2), 632-640. https/ / doi.org/ 10.1080/ 24748668.2015.11868820

Courel-Ibáñez, J., Sánchez-Alcaraz, B. . . \& Cañas, J. (2017). Game performanceandlength of rallyin professional padel players Journal of H uman Kintics, 55, 161- 169. https/ / doi.org/ 10.1515/ hukin2016-0045

Courel-Ibáñez, J., Sánchez-Alcaraz, B. J., García, S., \& Echegaray, M. (2017). Evolución del pádel en Españaenfunción del género yedad delospracticantes Cultura, Ciencia y Deporte, 12(34), 39-46. https/ / doi.org/10.12800/ ccd.v12i34.830

Courel-Ibáñez,J.,Sánchez-Alcarzz, B.J.,\& M uñoz, D. (2019). Exploring gamedynamicsin padel: implicationsfor assessmentand training Journal of Strength and Conditioning Research, 33(7), 1971- 1977. https:/ / doi.org/ 10.1519/ JSC.0000000000002126

Crespo, M., \& Reid, M. M. (2007). Motivationintennis, British Journal of SportsM ediane, 41(11), 769-772.

Fernández deO ssó,A.I (2019). Diseño deuna herramienta deanálisisde indicadoresderendimiento técnico-táctico en pádel: análisisy comparación en diferentesnivelesdejuego y sexo (Tesisdoctoral). U niversidad Pablo deO lavide, Sevilla

Field,A. (2018). Discovering statisticsusing IBM SPSS statistics(5th ed). SAGE PublicationsLtd.

Fritz, C. O., Morris, P.E., \& Richler,J.J. (2012). Effect sizeestimates: currentuse, calculations, and interpretation. Journal of Experimental Psychology: General, 141(1), 2-18.

Furlong, . . (1995).Theserviceinlawntennis: how importantisit?InT. Reilly, M. D. Hughes, \&A. Lees(Eds), Scienceand Racket Sports I. E\&FN Spon.

Gabin, B., Camerino, O.,Anguera, M.T., \&Castañer, M. (2012). Lince: multiplafform sportanalysissoftware. procedia Social and Behavioral Sciences, 46, 4692-4694. https:/ / doi.org/ 10.1016/ J.SBSPRO 2012.06 .320

GarcíaBenítez, S., Pérez-Billban,T., Echegaray,M., \& Felipe,J. L. (2016). Influenciadel género en laestructuratemporal y lasaccionesde juego del pádel profesional. Cultura, Cienciay Deporte, 11(33), 241247. https/ / doi.org/ 10.12800/ ccd.v11333.769

Gawin,W., Beyer, C., Hasse, H., \& Büsch, D. (2013). How to attackthe service: an empirica contribution to rally openingin world-class badminton doubles International Journal of PerformanceAnalysisin Sport, 13(3), 860-871.

Gillet, E., Leroy, D., Thouvarecq, R., \& Stein,J. F. (2009).A notational analysis of elitetennisserveand serve return strategies on slow surface. Journal of Strength and Conditioning Research, 23(2), 532539. https/ / doi.org/ 10.1519/ JSC.0b013e31818efe29

Gómez, M. Á., Lago-Peñas, C., \& Pollard, G. (2003). Situational varia bles. InT. McGarry,P.O'Donoghue, \&J. Sampaio(Eds), Routledge handbookofsportsperformanceanalysis(pp. 259-269). Routledge

Federación Internacional dePádel. (2017). Reglamento deJuego del Pádel. Recuperado el 1 de Septiembre de 2020: https: / www. padelfip.com/ es/ rules/ .

Federación Internacional dePádel. (2020). Listadepaisesasociadosala 
Federación Internacional dePádel (FIP). Recuperado el 1 deSeptiembrede2020, dehttps:/ / www. padelfip.com/ federations/.

Lupo, C., Condello, G., Courel-Ibánez, J., Gallo, C., Conte, D. , \& Tessitore, A. (2018). Effect of gender and match outcome on professional padel competition. RICYDE: Revista Internacional de CienciasDel D eporte, 14(51), 29-41. https:/ / doi.org/ 10.5232/ ricyde2018.05103

Martínez-Gallego, R., Crespo,M., Ramón-Llin,J., Micó,S., \& Guzmán, J.F. (2020). Men'sdoublesprofessional tennison hard courts: Game structureand point ending characteristics. Journal of H uman Sport and Exercise, 15(3), 633-642. doi:https:/ / doi.org/ 10.14198/ jhse.2020.153.13

Martínez, J.A. C., Moreno,J. F., \&Verdú, N. P. (2017).Análisisdela frecuencia cardíacaen el pádel femenino amateur. Retos N uevas Tendenciasen Educación Física, Deportey Recreación, (32), 204-207. https/ / doi.org 10.47197/ retos. V0i32.56040

Mesagno, C., Geukes, K., \& Larkin, P. (2015). Chokingunder pressure: A review of currentdebates, literature, and interventions In $S . D$. M ellalieu \& S. Hanton(Eds.), Contemporaryadvancesin sport psychology:A review NewYork: Routledge.

Muñoz, D., Courel-Ibáñez,J., Sánchez-Alcaraz, B., Dízz,.J.,Grijota,F.J. \& M uñoz,.J., (2017).Análisisdel uso y eficaciadel gl obo pararecuperar lared enfunción del contexto dejuego en pádel. Retos. N uevas Tendenciasen Educación Físca, Deportey Recreación, (31), 19-22. https/ / doi.org/ 10.47197/ retos. Wi31.48988

Muñoz, D., García,A., Grijota, F. J., Dízz, J., Sánchez, I. B., \& M uñoz,J. (2016). Influenciadeladuración del setsobrevariablestemporales dejuego en pádel. Apunts. Educacion Fiscaca y Deportes, 123, 69-75. https:/ / doi.org/ 10.5672/ apunts.2014-0983.es(2016/ 1).123.08

Muñoz, D., Sánchez-Alcarzz, B.J., Coure-lbáñez,J., Romero, E., Grijota, F. J., \& Diaz,.). (2016). Study about profileand distribution of padel courts in the Autonomous Community of Extremadura. EBalonmano.Com Revida deCienciasD el Deporte, 12(3), 223-230.

Muñoz, D., Toro-Román,V., Grijota, F.J., Courel-Ibáñez, J., SánchezPay,A., \& Sánchez-Alcaraz, B.J. (2021).Análisisantropométrico y desomatotipo enjugadoresdepádel enfunción desunivel dejuego. Retos. N uevasTendenciasen Educación Física, D eportey Recreación 41, 285-290. https/ / doi.org/ 10.47197/ retos. Vii41.84155.

O'Donoghue, P. (2001).Themostimportantpointsingrandslamsingles tennis. Research Quarterly for Exerciseand Sport, 72(2), 125-131. https/ / doi.org/ 10.1080/ 02701367.2001.10608942

O'D onoghue, P., \& Ballantyne,A. (2008).Theimpact of speed of service in Grand Slam singlestennis. InA. Lees, J. . Kahn, \& I. M aynard (Eds.), Scienceand Racket SportsIll (pp. 179-184).

O'Donoghue, P., \& Brown, E. (2009). Sequencesof servicepointsand themisperception of momentumin elitetennis. International Journal of PerformanceAnalyśsin Sport, 9(1), 113-127.

Pradas, F., Cachón, J., O tín, D., Q uintas,A.,Arraco,S. I.,\& Castellar, C. (2014).Anthropometric, physiologica and temporal andysisinelite femalepaddle players. Retos. NuevasTendenciasen Educación Físca, Deportey Recreación, 25, 107-122.

Priego, J. I., 0 laso, J., Llana, S., Pérez, P., Gonáez, J. C., \& Sanchís, M. (2013). Padel: aquantitativestudy of theshotsand movementsin thehigh-performance. Journal of Human Sport and Exercise, 8(4), 925-931. https/ / doi.org/ 10.4100/ jhse. 2013.84.04
Ramón-Llin,J., Guzmán,J. F., Llana, S., Martínez-Gallego, R., James, N., \&Vuèkoviæ, G. (2019). The effect of thereturn of serveon the server pair's movement parametersand rally outcomein padel using cluster analysis. Frontiersin Psychology,10(1194), 1-8. https/ / doi.org/ 10.3389/ fpsyg2019.01194

Ramón-Llin, J., Guzmán, J. F., Llana, S., Vuèkoviæ, G., \& James, N. (2013). Comparison of distance covered in paddlein the serve team according to performancelevel. Journal of $\mathrm{H}$ uman Sport and Exercise, 8(3), 738- 742. https:// doi. org/ 10.4100/ jhse 2013.8.Proc3.20

Ramón-Llin, J., Guzmán, J., Llana, S., Vuckovic, G., M uñoz, D., \& Sánchez-Alcaraz, B.J. (2021).Análisisdeladistanciarecorridaen pádel en función del nivel de juego y el número de puntos por partido. RetosN uevastendenciasEn Educacion Fisca D eportey Recreacion, 39, 205-209. https:/ doi.org/ 10.47197/ retos.V0i39.79322

Ramón-Llin,J., Guzmán,J.F., \& Martínez-Gallego, R. (2018). Compa ración delafrecuenciacardiacaen competición, entrejugadoresde pádel deelitey de categoríanacional. Retos N uevasTendenciasEn Educacion Fisca Deportey Recreacion, 33, 91- 95.

Ramón-Llin,J., Guzmán,J. F., Martinez-Gallego, R.,Vuèkoviæ, G., \& James, N. (2014). Time-motion analysis of pádel playersin two matches of the 2011 pro tour. In D. . Peter \& P. G. O 'D onoghue (Eds. ), PerformanceAnalysis of Sport IX. Routledge. https: / / doi.org 10.4324/9780203080443

Reid, M .,M cM urtrie, D., \&Crespo, M. (2010).Thereldionshipbetween match statisticsand top 100 rankingin professional men'stennis. International Journal of PerformanceAnalysisin Sport,10(2), 131- 138. https/ / doi.org/ 10.1080/ 24748668.2010.11868509

Sánchez-Alcaraz, B. J. (2013). Historiadel pádel. MaterialePara La His toria Del Deporte, 11, 57-60.

Sánchez-Alcaraz, B.J., J jiménez,V., M uñoz, D., \& Ramón-Llín,J. (2020, In press). Eficaciay distribución delosgolpesfinalistasdeataqueen pádel profesiona enfunción delazonadelapista. Revisal Internacional deM edicina y Cienciasdela Actividad Físca yel Deporte.

Sánchez-Alcaraz, B.J., Muñoz, D., Pradas, F., Ramón-Llín,J., Cañas,]., \& Sánchez-Pay, A. (2020). Analysis of serve and servereturn strategiesin elitemaleand femaleplayers. Applied Sciences, 10(19), 6693. https/ / doi.org/ 10.3390/ app10196693

Torres-Luque, G., Ramirez,A., Cabello-M anrique, D., Nikoladilis, P.T., \&Alvero-Cruz,J. R. (2015). Mach analysisof eliteplayersduring paddletenniscompetition. International Journal of PerformanceAnalysis in Sport, 15, 1135-1144. https:// doi. org/ 10.1080/ 24748668.2015.11868857

ZabalaLili,J., Gil, S. M., Badiola,A., Bidaurrazagaz Letona, I.,Vargas,A., \&Granados, C. (2016). Característicasfísicasy antropométricas, y análisisdejuego enjugadoresdepádel deelite. Búsqueda, 3(16), 3340.https/ / doi.org/ 10.21892/ 01239813.164 